\title{
USE OF HORIZONTAL AND VERTICAL EDGE PROCESSING TECHNIQUE TO IMPROVE NUMBER PLATE DETECTION
}

\author{
Soojey Deshpande ${ }^{1}$, Sandip Kamat ${ }^{2}$, Vaishali Patil ${ }^{3}$, Mukesh Patil ${ }^{4}$, Pradeep Patil ${ }^{5}$ \\ ${ }^{1}$ Research Scholar, Department of Electronics, JJT University, Rajasthan, India \\ ${ }^{2}$ Assistant Professor, Sinhgad Institute of Technology \& Science, SPPU, Pune, Maharashtra, India \\ ${ }^{3}$ Assistant Professor, Sinhgad Institute of Technology \& Science, SPPU, Pune, Maharashtra, India \\ ${ }^{4}$ Assistant Professor, Sinhgad Institute of Technology \& Science, SPPU, Pune, Maharashtra, India \\ ${ }^{5}$ Principal, KJ College of Engineering, SPPU, Pune, Maharashtra, India
}

\begin{abstract}
Automatic Number Plate Recognition (ANPR) is a bulk investigation system that catches the image of vehicles and identifies their license number. ANPR may be supported in the finding of taken vehicles. The recognition of taken vehicles may be done in an effective means by means of the ANPR systems situated on the highways. This paper proposes a recognition technique in which the vehicle plate image is found by the digital cameras and the image is processed to acquire the number plate data. A back image of a vehicle is taken and administered using numerous algorithms.
\end{abstract}

Key Words: Image Processing, Histogram, Skew Correction, Segmentation

\section{INTRODUCTION}

A new methodology in License Plate Recognition (LPR) system and associated algorithms proposed and experimentation with it is deliberate in this paper. This paper puts more emphasis on License Plate Detection from captured image of the vehicle. The database of over 600 images for this purpose is utilized for analysis.

\section{FLOW CHART OF ALGORITHM}

Each vehicle has an exclusive identity number on it License Plate. It is likely to identify this using Vehicle Registration Plate Identification System.

The consistency of the Vehicle Registration Plate Identification System depends upon its application. License plate recognition has limitations due to different circumstances, for example mist, shower, glooms, uneven illumination conditions, varying distances, the car's velocity, plate, tilt on frame, plate screw, number of vehicles in the frame, etc. These things make plate recognition very complicated and harder than the traditional systems [1] [2]. So we have discussed about how to implement an algorithm by means of the MATLAB for the Registration Plate Finding system.

MATLAB is a good alternative for the execution of algorithms which needs enormous computation, especially in Image Processing. MATLAB offers simple and fast employment of algorithms related to $\mathrm{C}$ and $\mathrm{C}++$. $\mathrm{A}$ considerable attribute in MATLAB has, it comprises a huge collection of Image Processing Functions and information investigation. Implementation and verification any algorithm with MATLAB is fast and reliable in advance to really employing this upon actual hardware. Recognition and errors were rectified, modifications in any algorithm on actual hardware system becomes a very difficult.

MATLAB offers an easy methodology for debugging, correction and modifications in any algorithm. MATLAB contains many for information investigation and image handling, so this one is a superior choice above other software languages for example $\mathrm{C} \& \mathrm{C}++$.

Considering all the above benefits, I have used MATLAB to implement my algorithm for Registration Plate Recognition [3]. This algorithm uses some inherent functions and some customized functions associated to image processing.

The algorithm was tested for many samples of car images. Once the process was fully confirmed, the inherent functions of MATLAB are exchanged with customized functions [4]. The flow chart in figure 1 shows the steps of execution of the procedure.

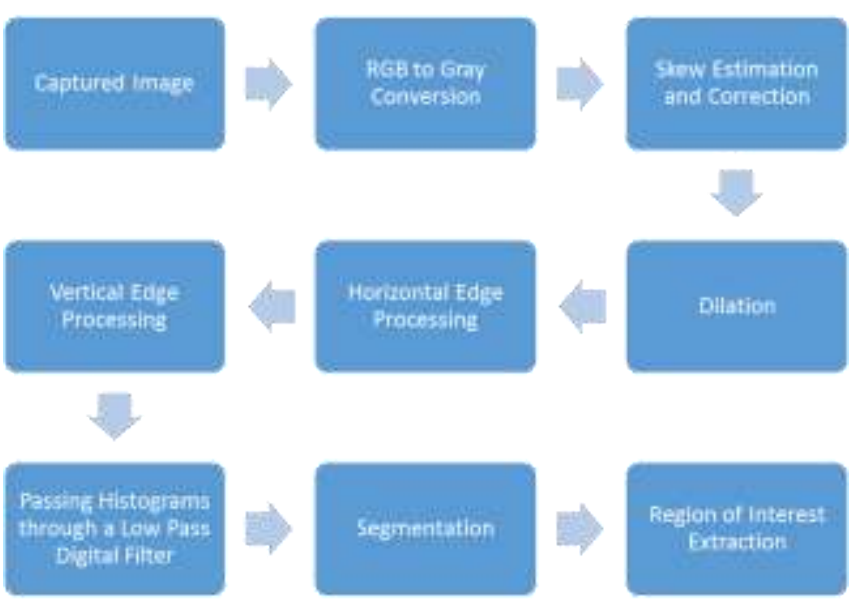

Fig -1: Flow chart of algorithm 


\subsection{Convert RGB Image into Gray Image}

The procedure explained here does not depend upon the sort of colors within an image, also depend mostly upon Gray Level of an image to manage and mining requisite data [5]. An RGB image have 3 frequencies: Red, Green \& Blue. RGB frequency coarsely tracks color Receptors within the Human Eye, and utilized by computer displays \& Image scanners.

Suppose the RGB image is 24-bit, each channel has 8-bits, in other words, this image is made of three images, each of every channel, in which every image may save distinct pixels by typical illumination strengths amongst 0 to 255 .

Suppose an RGB image [8] is 48-bit (excessive resolution), each channel is formed with 16-bit images. Color constituents such as Red, Green \& Blue values are not utilized all over this procedure. The input image is normally RGB. In advance processing, the brightness information is needed, so color images are transformed into grayscale images.

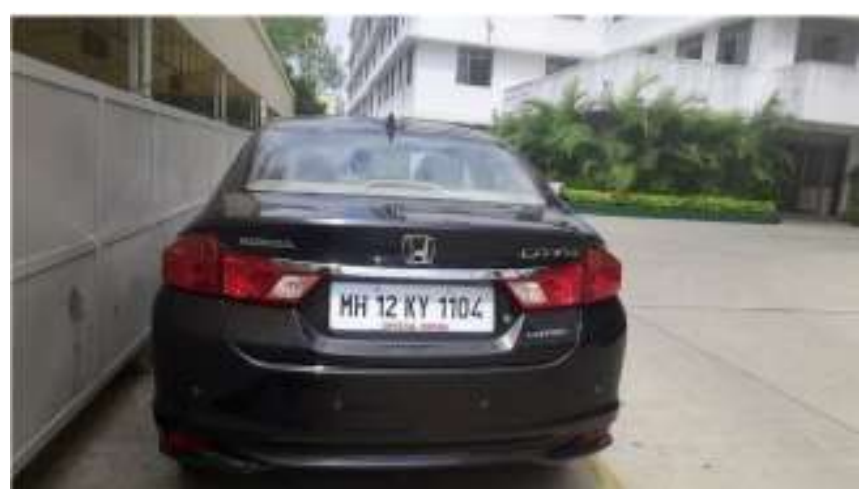

Fig -2: RGB input image

The RGB image has three constituents, Red, Green \& Blue pixels with fix strength. When an RGB image converted into gray image, then the strength of every pixel may be estimated from the pixel values of RGB image by using an equation.

$I_{Y}=0.2989 \times F_{R}+0.5870 \times F_{G}+0.1140 \times F_{B}-----$ (1)

Where $F_{R}, F_{G}$ and $F_{B}$ are the strengths of $\mathrm{R}, \mathrm{G} \& \mathrm{~B}$ constituent respectively, and $I_{Y}$ is the strength of similar gray level images of an RGB image.

Where $F_{R}, F_{G}$ and $F_{B}$ are the strengths of $\mathrm{R}, \mathrm{G} \& \mathrm{~B}$ constituent respectively, and $I_{Y}$ is the strength of similar gray level images of an RGB image.

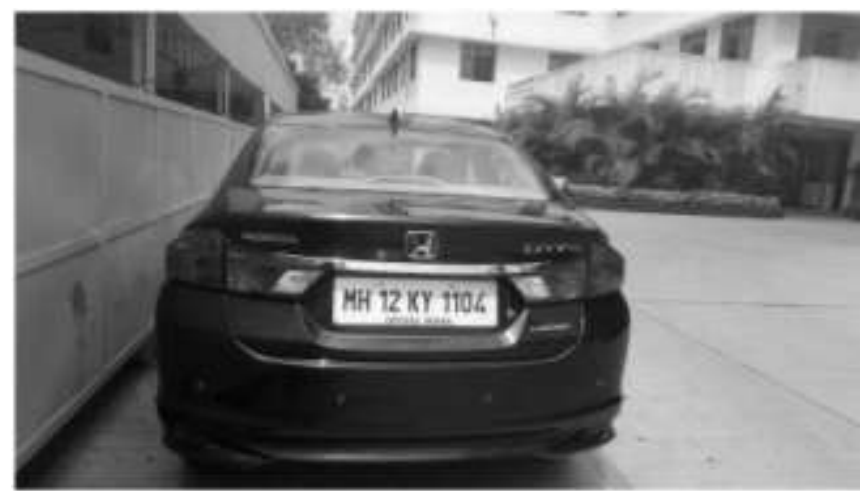

Fig -3: Gray image

\subsection{Skew Estimation and Correction}

The skewed License Plate affects severely on the precise character separation and identification [6]. To answer the problem, an effective technique for skew adjustment of License Plate is required. The skew estimation may be done using Radon Transfer. The skew rectification is done by turning the entered image by an angle $\theta$, which was acquired in the tilt estimation step.

Using the Radon Transform upon an image $\mathrm{f}(\mathrm{x}, \mathrm{y})$ for a certain number of angles may be computed the estimate of an image by the angles provided. A subsequent estimate is a summation of strengths of pixels within every direction, i.e. Line Integral. An outcome is image R (r,q).

This may be recorded mathematically by defining

$$
\rho=x \operatorname{Cs} \theta+y \sin \theta
$$

after which the Radon Transform may be recorded as

$$
R(\rho, \theta)=\int_{-\infty}^{\infty} \int_{-\infty}^{\infty} f(x, y,) \delta(\rho-x \cos \theta+y \sin \theta) d x d y
$$

In which, $\delta(\cdot)$ is the Dirac Delta Function.

An estimate of this function $f(x, y)$ is the certain number of "Line Integral". The Radon Function computes the Line Integrals from various sources along rays in a definite path. The rays are spread out one unit pixel apart. To signify an image, the Radon Function takes various similar ray predictions of image after several angles through turning this original about middle of the image.

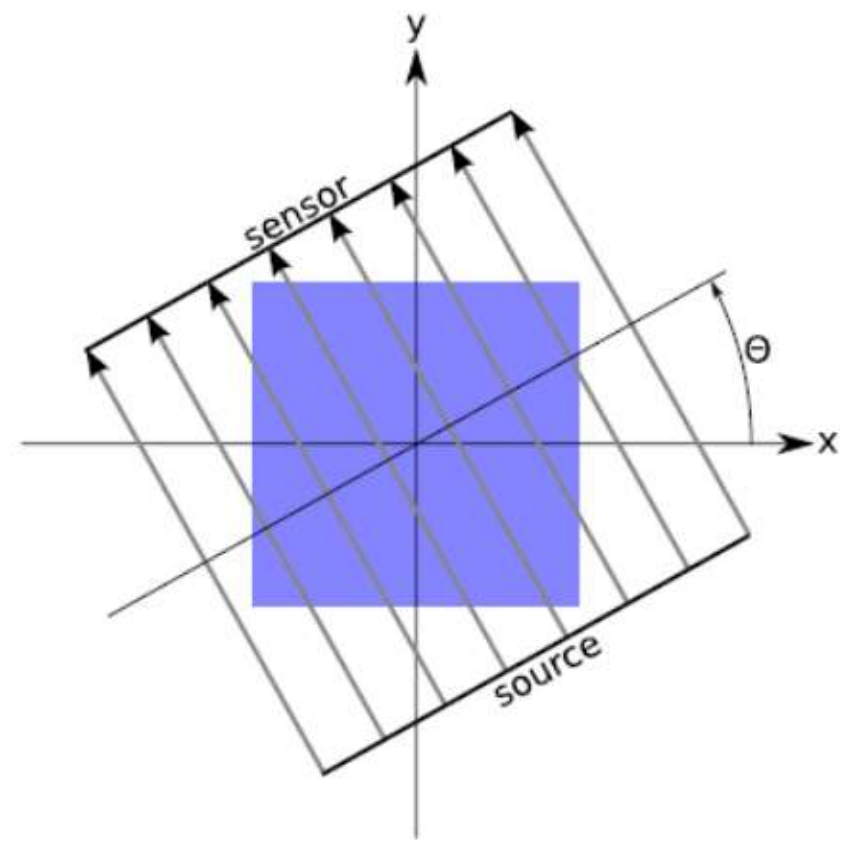

Fig -4: Object turned around the center of an object 


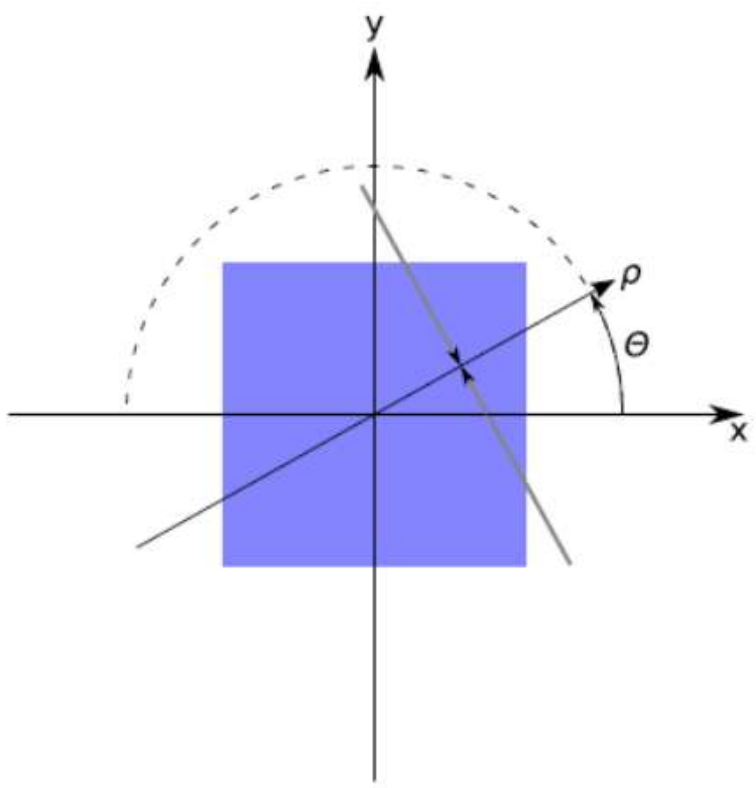

Fig -5: ${ }^{\boldsymbol{\rho}}$ axis crosses are summed up at the $R\left({ }^{\boldsymbol{\rho}}, \theta\right)$

The Source \& Sensor entrapment is turned around the midpoint of the body presented in figure 5 . For every angle $\rho$, the intensity of rays from source goes over is accrued next to the sensor. It is iterated by a given number of angels, typically after $\theta \in[0 ; 179]$.. The angle $180^{\circ}$ is not compressed, meanwhile result may be the same to angel $0^{0}$. From every angle $\theta$ and distance $\rho$ the intensity of the matter a ray vertical to the $\rho$ axis intersects are added up at the $\mathrm{R}(\rho, \theta)$ presented in figure 5. The white mark is the distance from the mid and the angle upon which the entire of strengths in the image summits. It is thus the angle formed by the line with the location.

To compute the Radon Transform of an image I for the angles indicated in the vector $\theta$, by the Radon Function with this composition.

$$
[R, x p]=\operatorname{radon}(I, \theta)
$$

The columns of $\mathrm{R}$ comprise the Radon Transform for every angle in $\theta$. The vector $x p$ comprises the equivalent coordinates by the $\mathrm{x}$-axis. Creating a matrix interpretation of the obtained lines and finding the angle of the highest noticeable line on the image, the skew angle is determined. The image is turned with the angle estimated as presented in figure 6.

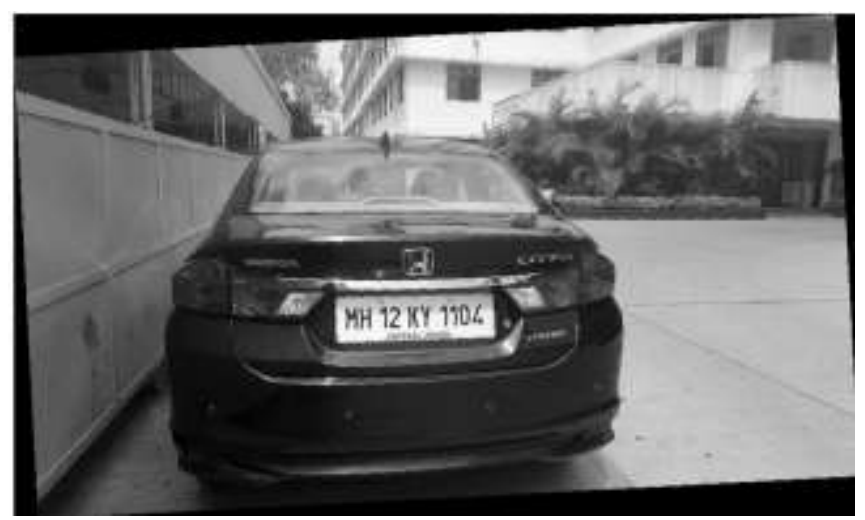

Fig -5: Image rotated by the angle estimated

\subsection{Dilation of an Image}

The method of Dilation [7] is an extemporizing certain image through loading Holes within an image, make edges of items within an image sharp, connect damaged lines to enhance image illumination. The amount of output pixel is the highest value of every pixel within entered the pixel's vicinity. In the Binary Image, if any, of the pixel values is made 1 , output pixel is made in 1 .

The fundamental outcome of the operator upon Grayscale Image is to expand the borders of areas of Foreground Pixels (i.e. Usually white pixels) gradually. Therefore, Foreground Pixels regions develop in dimension while holes inside those regions turn to tinier. Dilated Image is presented in figure 6.

On Grayscale Morphology, images are functions, charting a Euclidean Space/grid E into RU $\{\infty,-\infty\}$, where $\mathrm{R}$ is the set of reals, $\infty$ is a great component than all real numbers, and $\infty$ is a lesser component than all real numbers. Complete lattices were partly organized sets, in which each subset has an Infimum and a Supremum.

Especially, this comprises smallest component and largest component Grayscale organizing components are also functions of the similar layout and named as Structuring Functions. Signifying an image with $\mathrm{f}(\mathrm{x})$ and Structuring Function with $\mathrm{b}(\mathrm{x})$, Grayscale Dilation is given by

$$
(f \oplus b)(x)=\sup _{y \in E}[f(y)+b(x-y)]
$$

Where "sup" represents the supreme.

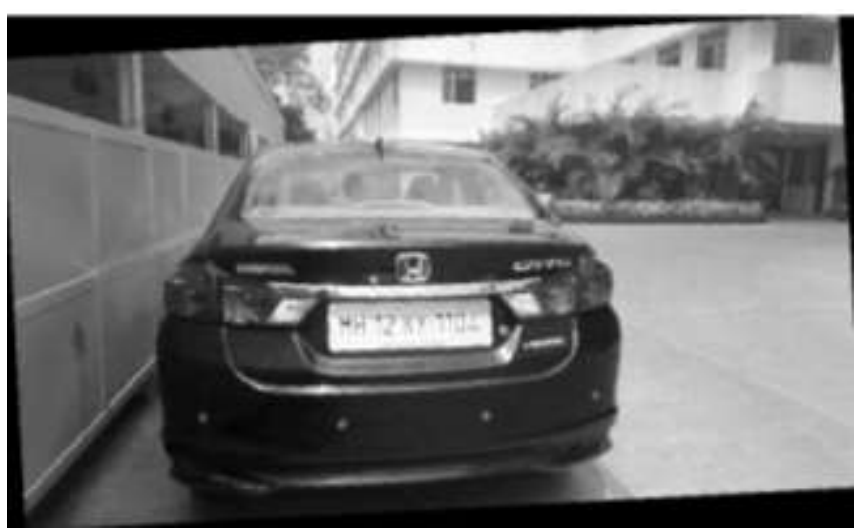

Fig -6: Dilated image

\subsection{Erosion of an Image}

The world of producing pixel is the smallest value among all pixels in entering the pixel's vicinity. In "Binary Image", if any, of the pixel value is made 0 , the output pixel is made in 0 .

Erosion is one crucial operator in area Mathematical Morphology, another is Dilation. It is usually employed on Binary Images, but various operations are performed upon Grayscale Images and consequently to finish matrixes. The elementary outcome of the operator on a Binary Image is to Erode the borders of areas of Foreground Pixels (i.e. Usually white pixels). Therefore, regions of Foreground Pixels 
contract in dimension, and holes in regions that turn out to be bigger. The figure 4.9 displays an Eroded Image.

When the constructing component B has midpoint (e.g., B is a circular or a rectangular), and the midpoint is situated upon the starting point of E, afterwards Erosion of A to B may be recognized as the curve of points extended with a midpoint of B when B travels within A. For an instance, Erosion of a rectangle of side 10, concentrated on starting point, by a circle of radius 2 , similarly concentrated on starting point, is a rectangle of side 6 concentrated on starting point.

Similarly, the Erosion of $\mathrm{f}$ by $\mathrm{b}$ is given by

$$
(f \ominus b)(x)=\inf _{y \in E}[f(y)-b(y-x)]
$$

The "Inf" means the Infimum.

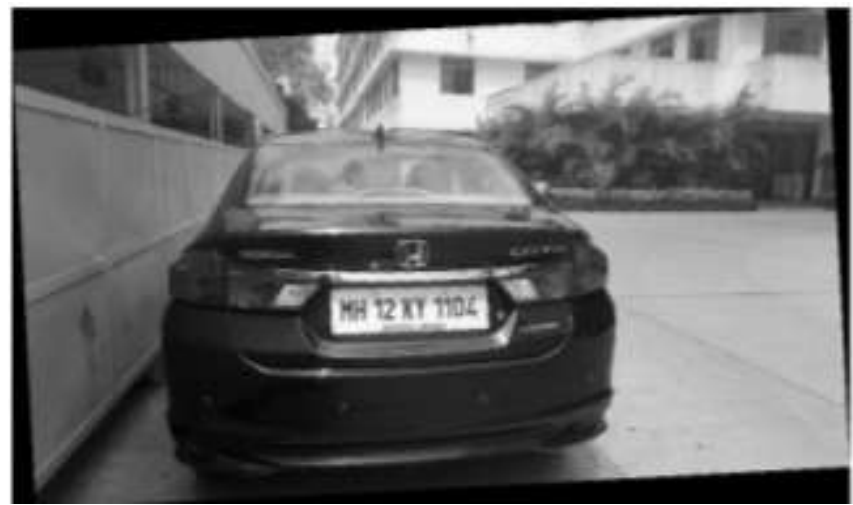

Fig -6: Dilated image

Grayscale Erosion with a flat diskette formed shaping component will usually blacken the image. Bright areas surrounded by black areas contract in dimensions, and black areas surrounded by bright areas enlarge in dimensions. Minor bright spots in images will vanish as they are Eroded away down to the neighboring strength value, and minor dim spots will turn into bigger spots. The outcome is maximum noticeable at spaces in the image where the intensity varies quickly and areas of moderately even intensity will be left comparatively unaffected excluding their boundaries.

\subsection{Horizontal and Vertical Edge Processing}

Boundaries resembled two huge breaks in the image. "Histogram" is a chart indicating quantities of a fluctuating amount above specified series. The Number Plate Detection Algorithm has employed Horizontal \& Vertical Histogram, that signifies column-wise \& row-wise Histogram correspondingly. Those Histograms signify an addition of variations in Gray Values amongst adjacent pixels of an image, column-wise \& row-wise. The Horizontal Histogram is computed initially within previous stage, presented in figure 4.10 .

To obtain a Horizontal Histogram, the procedure negotiates over every column of the image. In every column, procedure commences along second pixel after the topmost. Variance amongst 2nd and 1st pixel is computed. Suppose the variance go beyond specific verge, it is put into an entire edition of variances.

Then the procedure will shift down to compute the variance amongst the 3rd and 2nd pixels. This shifts on till termination of a column and compute entire addition of variances amongst adjacent pixels. Finally, an array comprising column-wise addition is formed. A similar procedure is performed to discover Vertical Histogram presented in figure 4.11. Within this event, rows are handled as a replacement of columns.

\subsection{Passing Histograms through a Low Pass Digital Filter}

Mentioning to diagrams displayed underneath, the Histogram estimates deviations severely amongst successive columns and rows. Hence, to avoid loss of significant data in forthcoming stages, this is desirable to flat out such excessive variations in the amounts of the Histogram.

To perform this the Histogram is processed using Low-pass Digital Filter. Low-Pass Filters are present in several dissimilar types, comprising Electronic Circuits, AntiAliasing Filters for acclimatizing images earlier to Analogto-Digital transformation, Digital Filters for leveling collections of information, Acoustic Barriers, Fogging of images, and so on.

Low-Pass Filters offer a flatter shape of a signal, eliminating the temporary variations, and leave behind the stretchedterm style. While the execution of this stage, average of every Histogram estimate considers the values upon its Right as well as Left side.

The stage makes this similarly upon Horizontal Histogram and Vertical Histogram. Diagrams beneath are displaying Histogram earlier without exceeding over Low-Pass Digital Filter thereafter with exceeding over Low-Pass Digital Filter.
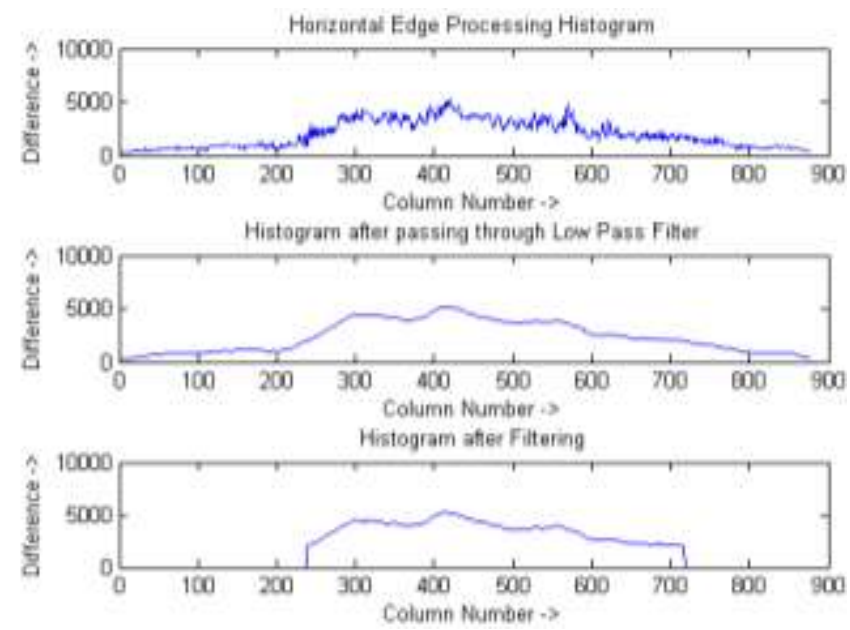

Fig -7: Horizontal Histogram 


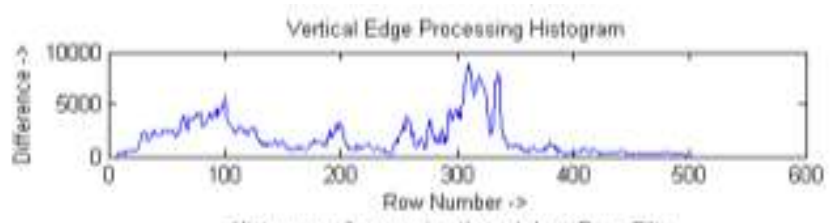

Histogram atter passing through Low Pass Filter

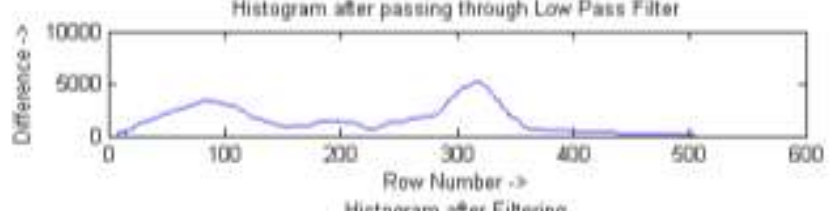

Histogram ater Filtering

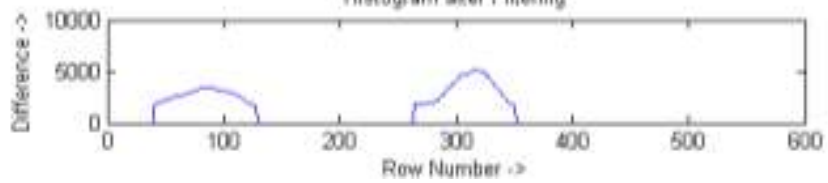

Fig -8: Vertical Histogram

When the Histograms are exceeded over Low-Pass Digital Filter, filter is employed for eliminating unwarranted regions of an image. Within the situation, unnecessary regions are Rows \& Columns have less Histogram values.

Less Histogram estimate specifies that portion of the image comprises extremely minute deviations between adjoining pixels. Meanwhile, an area having License Plate comprises simple environment with Alphanumeric Characters on that, the variance within adjacent pixels, specifically on the boundaries of Characters and Number Plate, might be extreme.

The outcome is an excessive Histogram value for such portion of an image. So, the area which has likely had License Plate, an extreme Horizontal \& Vertical Histogram estimates. Parts having the smallest value are therefore not essential any longer. Such parts are detached from an image by employing a Dynamic Threshold. In this procedure, the Dynamic Threshold is identical to the average value of a Histogram.

Individually Horizontal \& Vertical Histograms are succeeded over a filter by the Dynamic Threshold". The outcome of the procedure is Histogram displaying areas containing great possibility for comprising a Number Plate.

\subsection{Segmentation}

When License Plate has located, then the Segmentation is done [10]. A License Plate as explained above has excessive intensity deviation areas. The subsequent stage is to discover every portion of the areas within an image, which have great possibility for comprising a License Plate.

Co-ordinates of entire such possible areas were saved within an array. An effectual Character Segmentation is done by Line Scanning Technique. The obtained image showing the probable License Plate areas is displayed below in figure 9.

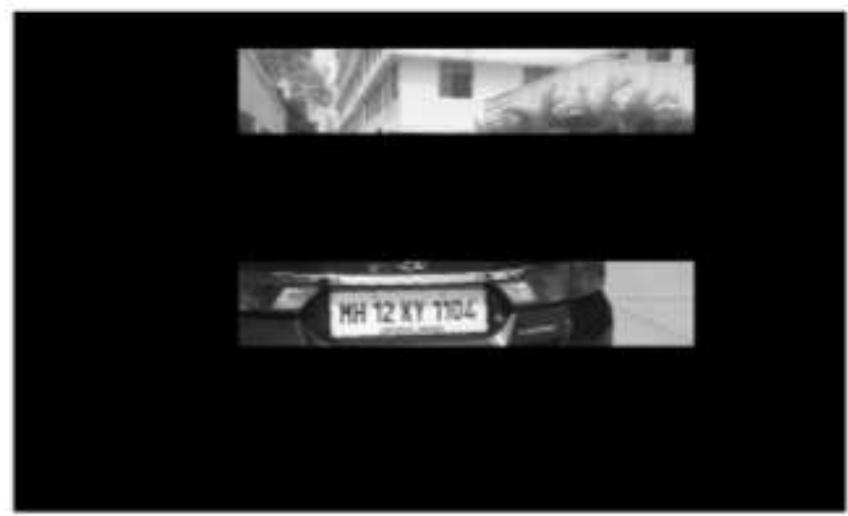

Fig -9: Probable License Plate areas

\subsection{Region of Interest Extraction}

The outcome of Segmentation procedure is every part of the area which possess highest possibility of compressing License Plate [9]. From those areas, one having the highest Histogram estimate is deliberate as the greatest possible aspirant of Number Plate.

The entire area is treated Row-wise \& Column-wise for discovering mutual areas containing the highest Horizontal \& Vertical Histogram worth. From those areas, one having the highest Histogram estimate is deliberate as the greatest possible aspirant of Number Plate [11]. An image discovered License Plate is presented in figure 10.

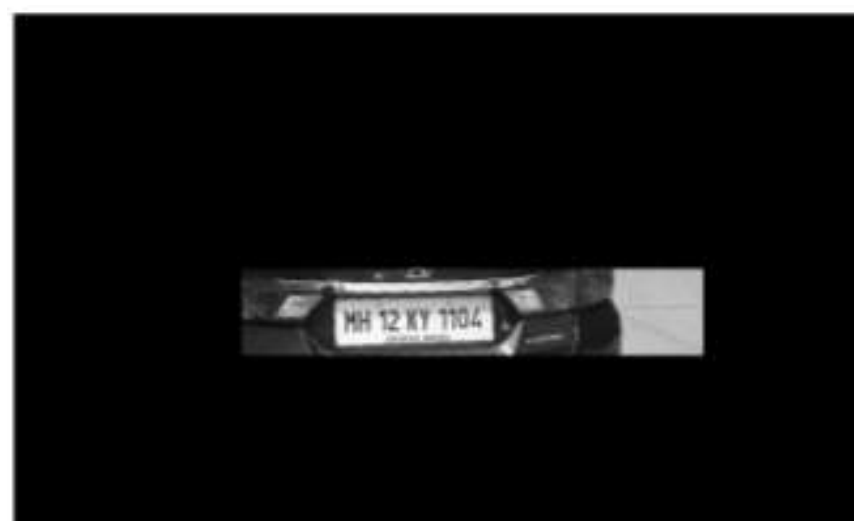

Fig -10: Location of License Plate

This procedure was confirmed by various intake images containing Resolution changing of $680 \times 480$ up to 1600 $\times 1200$ pixels. Images including vehicles with various colors as well as changing strength of illumination. Using this type of image, procedure properly identified Number Plate.

The same procedure had been tested upon images containing Number Plate associated with definite angle (appx. 8-100) at Horizontal Axis. Although by these types of images, Number Plates were identified effectively [12]. Erosion is identical to the procedure of Dilation, but at this juncture pixels were transformed into white. This destroys object borders as well as separates linkages, if the size of constituting component is larger than the joining pixel. 


\section{RESULTS}

The overall recognition rate of the CLPR system is measured here by considering the recognition of LP from three different views such as 2 Steps move from License Plate, 4 Steps move from License Plate and 6 Steps move from License Plate. Each of these three views have other three different viewpoints such as from straight view, one step in the left direction from the LP position and two steps in the left direction from the LP position. Thus totally nine different viewpoints are obtained as illustrated in the figure 11 .

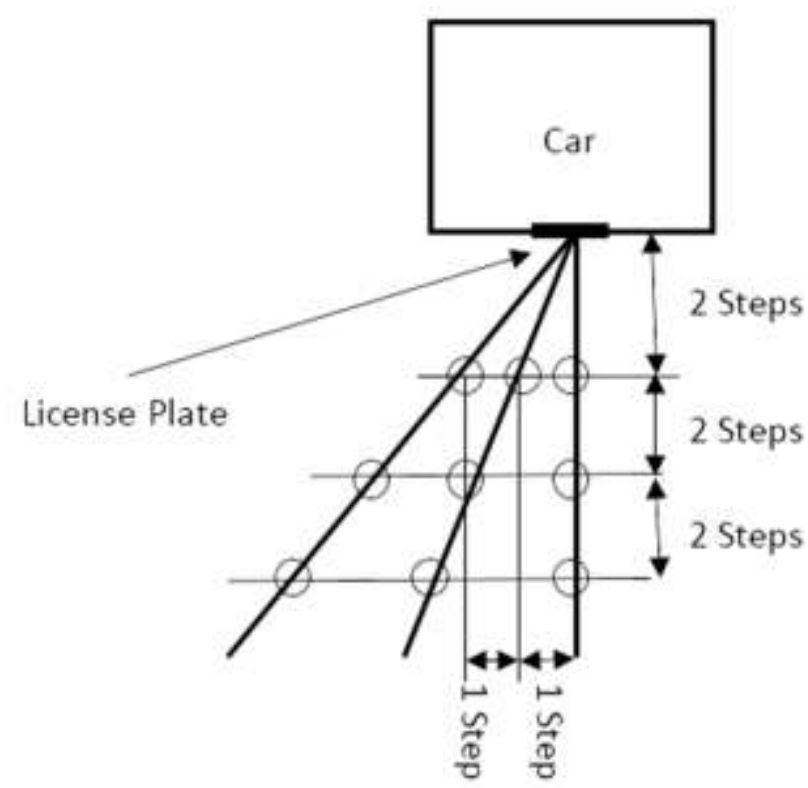

Fig -11: View Points of License Plate (1 Step=60 c.m.)

The variables used for denoting different viewpoints mentioned above are tabulated in the table 1

Table 1. Different viewpoints of LP

\begin{tabular}{|l|l|l|l|}
\hline $\begin{array}{l}\text { View Point of License } \\
\text { Plate }\end{array}$ & Straight & $\begin{array}{l}\text { 1 Step } \\
\text { Left }\end{array}$ & $\begin{array}{l}\text { 2 steps } \\
\text { Left }\end{array}$ \\
\hline $\begin{array}{l}\text { 2 Steps away from } \\
\text { License Plate }\end{array}$ & $\mathrm{X} 1$ & $\mathrm{Y} 1$ & $\mathrm{Z} 1$ \\
\hline $\begin{array}{l}\text { 4 Steps away from } \\
\text { License Plate }\end{array}$ & $\mathrm{X} 2$ & $\mathrm{Y} 2$ & $\mathrm{Z} 2$ \\
\hline $\begin{array}{l}\text { 6 Steps away from } \\
\text { License Plate }\end{array}$ & $\mathrm{X} 3$ & $\mathrm{Y} 3$ & $\mathrm{Z} 3$ \\
\hline
\end{tabular}

\subsection{License Plate Localization Efficiency}

The License Plate Localization efficiency can be measured as the relation of the quantity of License Plates located correctly in connection with the total quantity of License Plates. It is given by the equation

$\%$ License Plate localization efficiency $=\frac{\text { Successful samples }}{\text { Total no of samples }} \times 100$

The License Plate Localization Efficiency may be measured by the above equation for the various viewpoints and the results are charted in the table 2 .
Table 2. License Plate Localization Efficiency measurement readings

\begin{tabular}{|l|c|c|c|}
\hline View Point & X1 & X2 & X3 \\
\hline Sample Images & 74 & 62 & 53 \\
\hline Located License Plates & 74 & 62 & 53 \\
\hline *Efficiency (\%) & 100 & 100 & 100 \\
\hline
\end{tabular}

\begin{tabular}{|l|c|c|c|}
\hline View Point & Y1 & Y2 & Y3 \\
\hline Sample Images & 60 & 60 & 61 \\
\hline Located License Plates & 60 & 60 & 61 \\
\hline *Efficiency (\%) & 100 & 100 & 100 \\
\hline
\end{tabular}

\begin{tabular}{|l|c|c|c|}
\hline View Point & $\mathbf{Z 1}$ & $\mathbf{Z 2}$ & $\mathbf{Z 3}$ \\
\hline Sample Images & 62 & 62 & 60 \\
\hline Located License Plates & 62 & 62 & 58 \\
\hline *Efficiency (\%) & 100 & 100 & 96.67 \\
\hline
\end{tabular}

\begin{tabular}{|l|c|}
\hline View Point & Total Samples \\
\hline Sample Images & 554 \\
\hline Located License Plates & 552 \\
\hline *Efficiency (\%) & 99.63 \\
\hline
\end{tabular}

\section{CONCLUSIONS}

For locating the "License Plate" area, there must be an accurate edge concerning to the "License Plate" border along with the car within the background, for that "Sobel Operator" is employed to discover the edge.

\section{REFERENCES}

[1] Choudhury A. Rahman, Wael Badawy, Ahmad Radmanesh,"A real time vehicle's license plate recognition system", Proceedings of IEEE Conference on Advanced Video and Signal Based Surveillance (AVSS'03). 2003

[2] S. R. Deshpande, Dr. P. M. Patil," License Plate Recognition using Fuzzy Neural Approach", in IEEE Bangalore Section 20th Annual Symposium on Emerging Applications of ICT in Utilities, November, 2011.

[3] Kumar Parasuraman, "An Efficient Method for Indian Vehicle License Plate Extraction and Character Segmentation", 2010 IEEE International Conference on Computational Intelligence and Computing Research, ISBN: 9788183713627

[4] Shyang-Lih Chang, Li-Shien Chen, Yun-Chung Chung, andSei-Wan Chen," Automatic license plate recognition", IEEE Transactions on Intelligent Transportation Systems, Vol.5, No.1, March 2004.pp. 44-53 
[5] VidyaSudhan L Nathan, Ramkumar J, KamakshiPriya S," New approaches for license plate recognition system", International Conference on Intelligent sensing and Information Processing (ICISIP 2004), pp. 149-152.

[6] Amr Badr, Mohamed M. Abdelwahab, Ahmed M. Thabet, and Ahmed M. Abdelsadek,"Automatic license plate recognition", Annals of the University of Craiova, Mathematics and Computer Science Series Volume 38 (1), 2011, Pages 62 \{71ISSN: 1223-6934

[7] F.A. Silva, A.O. Artero, M.S.V. Paiva3and R.L. Barbosa," ALPRS - A New Approach for License Plate", Signal \& Image Processing: An International Journal (SIPIJ) Vol.4, No.1, February 2013

[8] Yingjun $\mathrm{Wu}$, Shouxun Liu and Xuan Wang," License Plate Location Method Based on Texture and Color", Software Engineering and Service Science (ICSESS), 2013 4th IEEE International Conference on 23-25 May 2013, 978-1-4673-5000-6/13/\$31.00 @2013 IEEE

[9] Ashim Kumar Ghosh, Swapan K.D. Sharma, Md. Nazrul Islam, Suchitra Biswas, SameyaAkter," Automatic License Plate Recognition (ALPR) for Bangladeshi Vehicles"

[10] M. M. Shidore, S. P. Narote," Number Plate Recognition for Indian Vehicles”, IJCSNS International Journal of Computer Science and Network Security, VOL.11 No.2, Feb. 2011

[11] DhawalWazalwar, ErdalOruklu, JafarSaniie," A Design Flow for Robust License Plate Localization and Recognition in Complex Scenes", Journal of Transportation Technologies, 2012, 2, 13-21

[12] S. R. Deshpande, Dr. P. M. Patil," Implementation of Vehicle License Plate Detection Algorithm in MATLAB", the Board of International Journal of Latest Technology in Engineering Management \& Applied Science, Volume III, Issue IV, April 2014, ISSN 2278 - 2540. 